\title{
A syndrome of congenital microcephaly, intellectual disability and dysmorphism with a homozygous mutation in FRMD4A
}

\author{
Dina Fine ${ }^{1,8}$, Hagit Flusser ${ }^{2,8}$, Barak Markus ${ }^{1}$, Zamir Shorer ${ }^{3}$, Libe Gradstein ${ }^{4}$, Shareef Khateeb ${ }^{1}$, Yshia Langer ${ }^{1}$, \\ Ginat Narkis ${ }^{1}$, Ruth Birk ${ }^{5}$, Aharon Galil ${ }^{2}$, Ilan Shelef ${ }^{6}$ and Ohad S Birk ${ }^{\star, 1,7}$
}

A consanguineous Bedouin Israeli kindred presented with a novel autosomal recessive intellectual disability syndrome of congenital microcephaly, low anterior hairline, bitemporal narrowing, low-set protruding ears, strabismus and tented thick eyebrows with sparse hair in their medial segment. Brain imaging demonstrated various degrees of agenesis of corpus callosum and hypoplasia of the vermis and cerebellum. Genome-wide linkage analysis followed by fine mapping defined a $7.67 \mathrm{Mb}$ disease-associated locus (LOD score 4.99 at $\theta=0$ for marker D10S1653). Sequencing of the 48 genes within the locus identified a single non-synonymous homozygous duplication frameshift mutation of 13 nucleotides (c.2134_2146dup13) within the coding region of FRMD4A, that was common to all affected individuals and not found in 180 non-related Bedouin controls. Three of 50 remotely related healthy controls of the same tribe were heterozygous for the mutation. FRMD4A, member of the FERM superfamily, is involved in cell structure, transport and signaling. It regulates cell polarity by playing an important role in the activation of ARF6, mediating the interaction between Par3 and the ARF6 guanine nucleotide exchange factor. ARF6 is known to modulate cell polarity in neurons, and regulates dendritic branching in hippocampal neurons and neurite outgrowth. The FRMD4 domain that is essential for determining cell polarity through interaction with Par3 is truncated by the c.2134_2146dup13 mutation. FRMD4A polymorphisms were recently suggested to be a risk factor for Alzheimer's disease. We now show a homozygous frameshift mutation of the same gene in a severe neurologic syndrome with unique dysmorphism. European Journal of Human Genetics (2015) 23, 1729-1734; doi:10.1038/ejhg.2014.241; published online 12 November 2014

\section{INTRODUCTION}

The molecular defects leading to many of the intellectual disabilitymicrocephaly human phenotypes are yet to be deciphered. ${ }^{1}$ Consanguineous inbred-extended families are at high risk for autosomal recessive diseases, and are conducive to elucidation of the molecular basis of such diseases. The Bedouin community of southern Israel is extremely inbred within tribes, leading to abundance of monogenic autosomal recessive disorders. ${ }^{2}$ We now describe an extended Bedouin kindred afflicted with a novel congenital microcephaly intellectual disability syndrome with agenesis of corpus callosum and/or partial hypoplasia of the vermis and cerebellum, that is accompanied by unique dysmorphism. We demonstrate that this novel human phenotype is associated with a mutation in FRMD4A.

\section{MATERIAL AND METHODS}

Autozygosity mapping

Following Soroka Medical Center IRB approval and informed consent, genome-wide linkage analysis was done using GeneChip human mapping $250 \mathrm{~K}$ SNP arrays followed by fine mapping using polymorphic markers, as previously described. ${ }^{3}$ LOD scores were calculated using SUPERLINK (Haifa, Israel). ${ }^{4}$

\section{DNA sequencing}

Sanger sequencing was done using ABI 3130XL DNA analyser (Applied Biosystems, Rhenium Modi'in, Israel). Whole-exome sequencing was performed by HiSeq2000 using paired-end $(2 \times 100)$ protocol at a mean coverage of 30 -fold $(85-90 \%$ of all exonic nucleotides covered by $>10$ reads). For exome enrichment we used NimbleGen SeqCap EZ Human Exome Library v2.0 (Roche, Basel, Switzerland) targeting $44.1 \mathrm{Mb}$ regions. Sequencing read alignment, variant calling and annotation was performed by DNAnexus (DNAnexus Inc., Mountain View, CA, USA dnanexus.com). Filtering for known variants was done sifting through dbSNP database and Exome Variant Server, NHLBI Exome Sequencing Project, Seattle, WA, USA; accessed December 2011) and through our local databank of normal Bedouin controls. ${ }^{5}$ The identified variant was submitted to the Leiden Open Variation Database: http://databases.lovd.nl/shared/genes/ FRMD4A

\section{BseYI restriction analysis}

Analysis of the c.2134_2146dup13 (NM_018027.3) mutation was performed by differential BseYI restriction analysis: the mutation generated a novel BseYI restriction site leading to differential cleavage products of the mutant (98 and $79 \mathrm{bp}$ ) versus wild type (164 bp)

\footnotetext{
${ }^{1}$ The Morris Kahn Laboratory of Human Genetics, National Institute for Biotechnology in the Negev and Faculty of Health Sciences, Ben-Gurion University of the Negev, Beer-Sheva, Israel; '2Zusman Child Development Center, Pediatric Division, Soroka University Medical Center and Ben-Gurion University of the Negev, Beer-Sheva, Israel; ${ }^{3}$ Pediatric Neurology unit, Pediatric Division, Soroka University Medical Center and Ben-Gurion University of the Negev, Beer-Sheva, Israel; ${ }^{4}$ Department of Ophthalmology, Soroka University Medical Center and Clalit Health Services, Ben-Gurion University of the Negev, Beer-Sheva, Israel; ${ }^{5}$ Faculty of Health Sciences, Department of Nutrition, Ariel University, Ariel, Israel; ${ }^{6}$ Department of Diagnostic Imaging, Soroka Medical Center, Beer-Sheva, Israel; ${ }^{7}$ Soroka Medical Center, Genetics Institute, Beer-Sheva, Israel

${ }^{8}$ These authors contributed equally to this work.

${ }^{*}$ Correspondence: Professor OS Birk, Soroka Medical Center, Genetics Institute, Beer-Sheva, Israel. Tel: +972 8 6403439; Fax: +972 8 6400042; E-mail: obirk@bgu.ac.il

Received 24 May 2014; revised 13 August 2014; accepted 30 September 2014; published online 12 November 2014
} 
alleles. PCR amplification primers: 5' -gcagGTCGGTGGACATCAG-3' and 5'-GTCTGAGCCGTTGCTGCTA-3'.

\section{RESULTS}

\section{Clinical details}

Six individuals of consanguineous inbred Israeli Bedouin kindred (Figure 1a) presented with a novel apparently autosomal recessive syndrome: severe congenital microcephaly, intellectual disability, hypertonia at birth that lessened with age, and unique dysmorphism (Figure 2a and $\mathrm{b}$ ): hirsutism, low anterior hairline and bitemporal narrowing, low-set posterior rotated protruding ears, strabismus and fleshy slightly protruding lower lip, slightly anteverted nares with a normal philtrum; eyebrows were arched and thick with sparse hair in their medial segment and there was lateral puffiness above the upper eyelids and a skin fold partially covering the inferior eyelids, as well as long eyelashes. In terms of growth curves (see Table 1): all were born at term with birth weights adequate for gestational age (except one case of mild SGA). Head circumference was below third percentile in all at birth and throughout follow-up until 7 years of age. Weight and height were between less than third percentile and up to tenth percentile. All patients had different levels of ataxia, evident as early as 18 months of age. Intellectual disability was severe, as demonstrated in the affected individuals that were systematically evaluated (Table 1): four of the patients were evaluated at age 3 years (Vineland adaptive behavior scale), demonstrating developmental capabilities appropriate for ages 5-9 months. At 7 years of age, three of the four individuals were evaluated again: two were wheel chair bound (one of whom could stand assisted) whereas the third patient walked independently. Cerebellar signs were evident on neurological exam: uncoordinated hand movements were seen, and the only patient who walked independently had severe ataxia. Two of the three patients evaluated at 7 years did not speak at all and the third had a total vocabulary of two words. Fine motor capabilities were adequate for less than 1 year of age (could return object to box). Brain imaging in all four affected individuals tested, demonstrated partial to near full agenesis of corpus callosum and various degrees of hypoplasia of the vermis and cerebellum (Figure 2c), evident already in utero (Figure 2d). Karyotype was normal. Parents and unaffected siblings of affected individuals had no abnormal phenotype.

\section{Autozygosity mapping}

Genome-wide homozygosity mapping, testing five affected individuals and two unaffected parents, identified three candidate regions of homozygous haplotypes shared by the affected individuals. However, fine mapping, studying the entire kindred using polymorphic markers ruled out two of the loci (data not shown). The remaining single disease-associated $7.67 \mathrm{Mb}$ locus (Figures $1 \mathrm{~b}$ and c) was on chromosome $10 p$ between markers ch10.11922Kb and D10S466 with a maximal multi-point LOD score of $4.9944(\theta=0)$ for marker D10S1653 (data not shown).

\section{Sanger and whole-exome DNA sequencing}

Sanger sequencing of 30 of the 48 candidate genes within this locus identified a single non-synonymous homozygous duplication mutation of 13 nucleotides (c.2134_2146dup13, NM_018027.3) in the coding region of FRMD4A (Figure $3 \mathrm{a}-\mathrm{d}$ ), that was common to all affected individuals and not found in our whole-exome data bank of Bedouin controls. Whole-exome sequencing of an affected individual (Figure 1a, individual IV-3) identified no further homozygous nonsynonymous novel variants within the defined locus that were not found in healthy controls. No mutations were found in genes NIPBL, SMC1A and SMC3.

\section{Mutation analysis}

Analysis of the c.2134_2146dup13 mutation in the entire family and control samples was performed by differential BseYI restriction analysis: the FRMD4A mutation segregated within the family as expected, and was not found in any of 180 non-related healthy Bedouin controls of other tribes (data not shown). Within the tribe of the affected kindred, excluding the specific families depicted in Figure 1a, 3 of 50 controls were found to be heterozygous mutation carriers and none were found to be homozygous.

At the protein level, the duplication mutation results in a frameshift, leading to missense coding beginning at amino acid 716 and an early stop codon (p.Gly716Profs ${ }^{\star} 25$ ) generating a putative 740 amino acid protein in place of the native 1039 amino-acid protein (Figure 3e). ClustalW software analysis of FRMD4A protein sequences of different species (HomoloGene database) demonstrated significant amino-acid conservation at the serine-rich domain abrogated by the mutation (data not shown).

\section{DISCUSSION}

The novel dysmorphism, microcephaly and intellectual disability syndrome we describe has some features reminiscent of Cornelia de Lange syndrome, such as hirsutism, arched eyebrows and long eyelashes, as well as the increased muscle tone and the growth retardation and microcephaly. However, other clinical features, as well as the brain imaging indicate that the syndrome described here is a distinct novel one. Moreover, whole-exome sequencing of an affected individual failed to identify mutations in NIPBL, SMC1A and SMC3 genes known to be associated with Cornelia de Lange. With a FRMD4A mutation carrier frequency of $3 / 50$ in a tribe of approximately 2000 individuals, carrier testing and prenatal diagnosis can be utilized in disease prevention.

FRMD4A is localized to the cytoplasm and the cytoskeleton, and binds molecules in the undercoat of the cell-to-cell adherens junction. ${ }^{6,7}$ Its protein sequence has similarity to the highly related proteins 4.1 protein, Ezrin, radixin and moesin, comprising the FERM protein superfamily. ${ }^{7,8}$ This superfamily includes ubiquitous components of the cytocortex involved in cell structure, transport and signaling functions. ${ }^{6}$ FERM proteins contain three domains: the FERM domain, a central helical domain and a C-terminal tail domain, which binds F-actin. The FERM domain, located at the $\mathrm{N}$ terminus of the majority of FERM-containing proteins ${ }^{7,8}$ is responsible for cytoskeleton-membrane cross-linking: it attaches to the membrane by binding specific membrane proteins, whereas the last 34 residues of the C-terminal tail domain bind actin filaments. ${ }^{7,8}$ Aside from binding to membranes, the activated FERM domain can also bind the guanine nucleotide dissociation inhibitor of Rho GTPase, which suggests that in addition to functioning as cross-linkers, FERM proteins may influence Rho signaling pathways. ${ }^{8}$

In epithelial cells, FRMD4A was shown to regulate cell polarity by connecting ARF6 activation with the PAR complex (Par3/Par6/aPKC/ Cdc42), known to function in various cell polarization events. ${ }^{6,7}$ Activation of ARF6, a central player in actin cytoskeleton dynamics and membrane trafficking, is spatiotemporally regulated as a downstream signaling pathway of the Par protein complex: ${ }^{8,9}$ activation of ARF6 requires activity of guanine nucleotide exchange factors (GEFs), proteins exchanging GDP for GTP. ${ }^{9}$ Activation of ARF6 in the process of epithelial polarization is restrictively controlled via Cytohesin family proteins: Cytohesin1 is the GEF for ARF6, stimulating guanine 
a

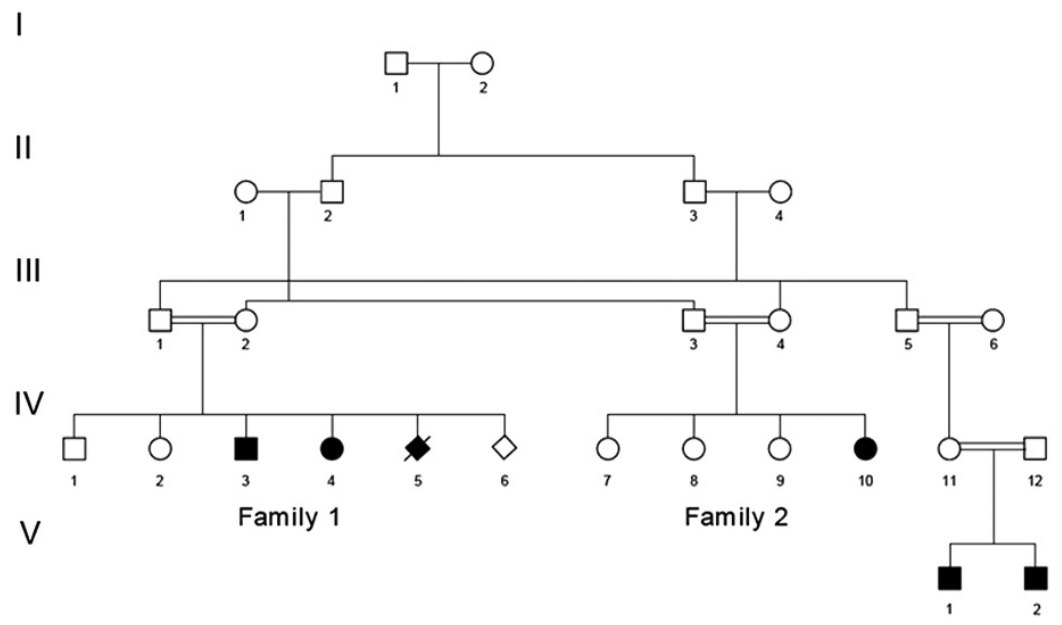

Family 3

b

Family 1

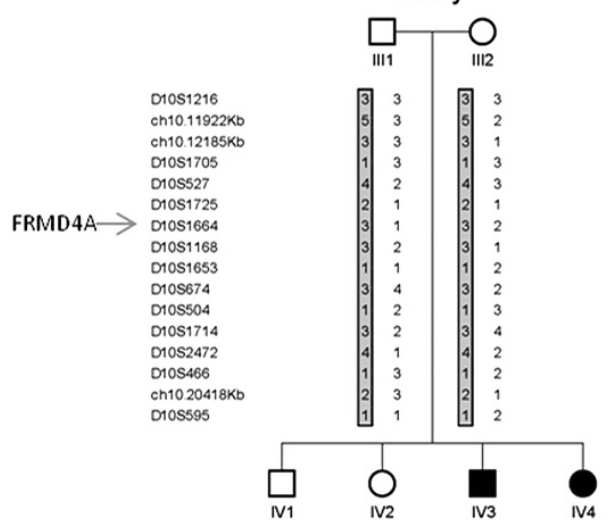

Family 2

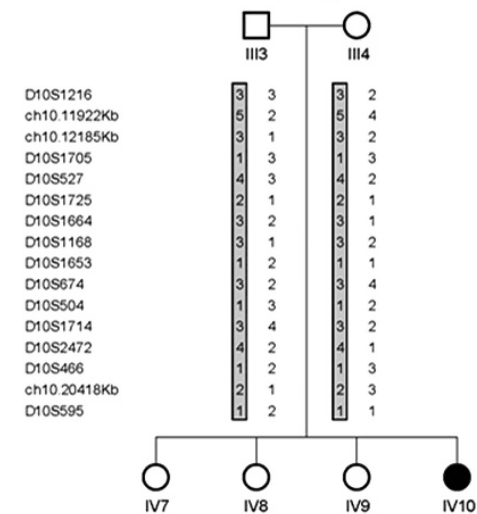

Family 3

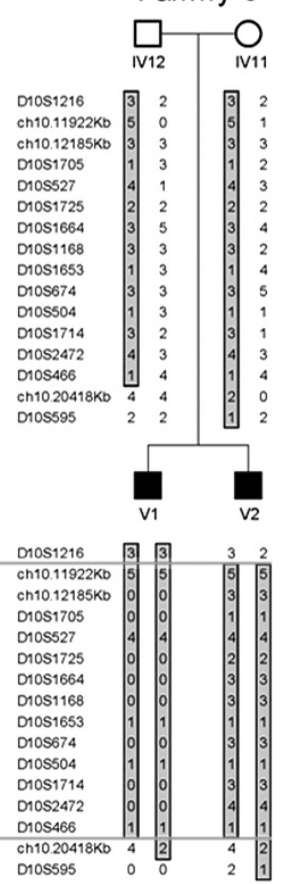

c
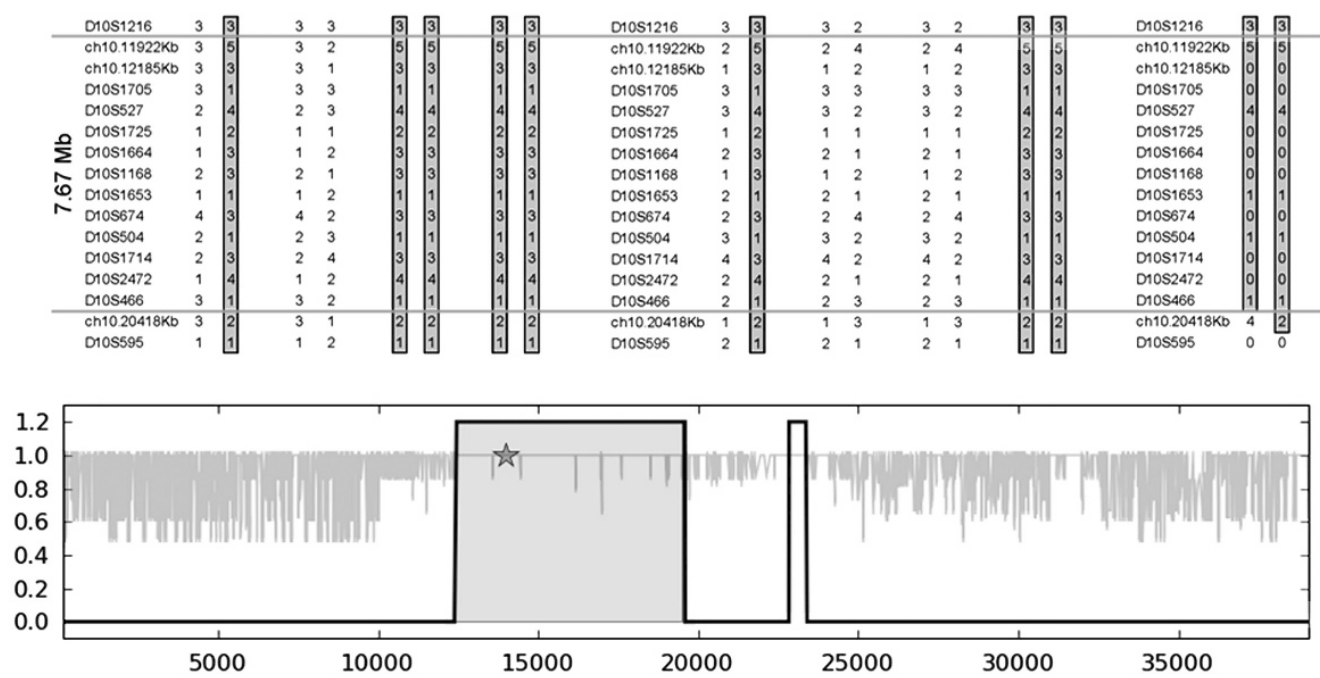

Figure 1 The affected kindred and fine mapping of the chromosome 10p locus: (a) Family tree of the affected kindred. (b) Fine mapping of the diseaseassociated locus. Haplotype of the locus in linkage with the disease. The disease-associated haplotype is boxed, and the minimum homozygous haplotype shared by all the affected individuals and by their obligatory carriers is defined by the red lines, markers ch10.11922Kb and D10S466. (c) Location of the mutated gene within the disease-associated locus (output of the homozygosity mapping tool for part of chromosome 10). The analysis shows the level of allele sharing between all affected individuals in gray lines (between 0 and 1 ). Confirmed shared blocks for all affected individuals are indicated by the thick black line. Within the regions, a few single-nucleotide polymorphisms are not homozygous due to background noise which reflects the error rates in the data. The highlighted region shows the disease-associated locus and the red star marks the location of the mutated gene, FRMD4A. The full colour version of this figure is available at European Journal of Human Genetics online. 

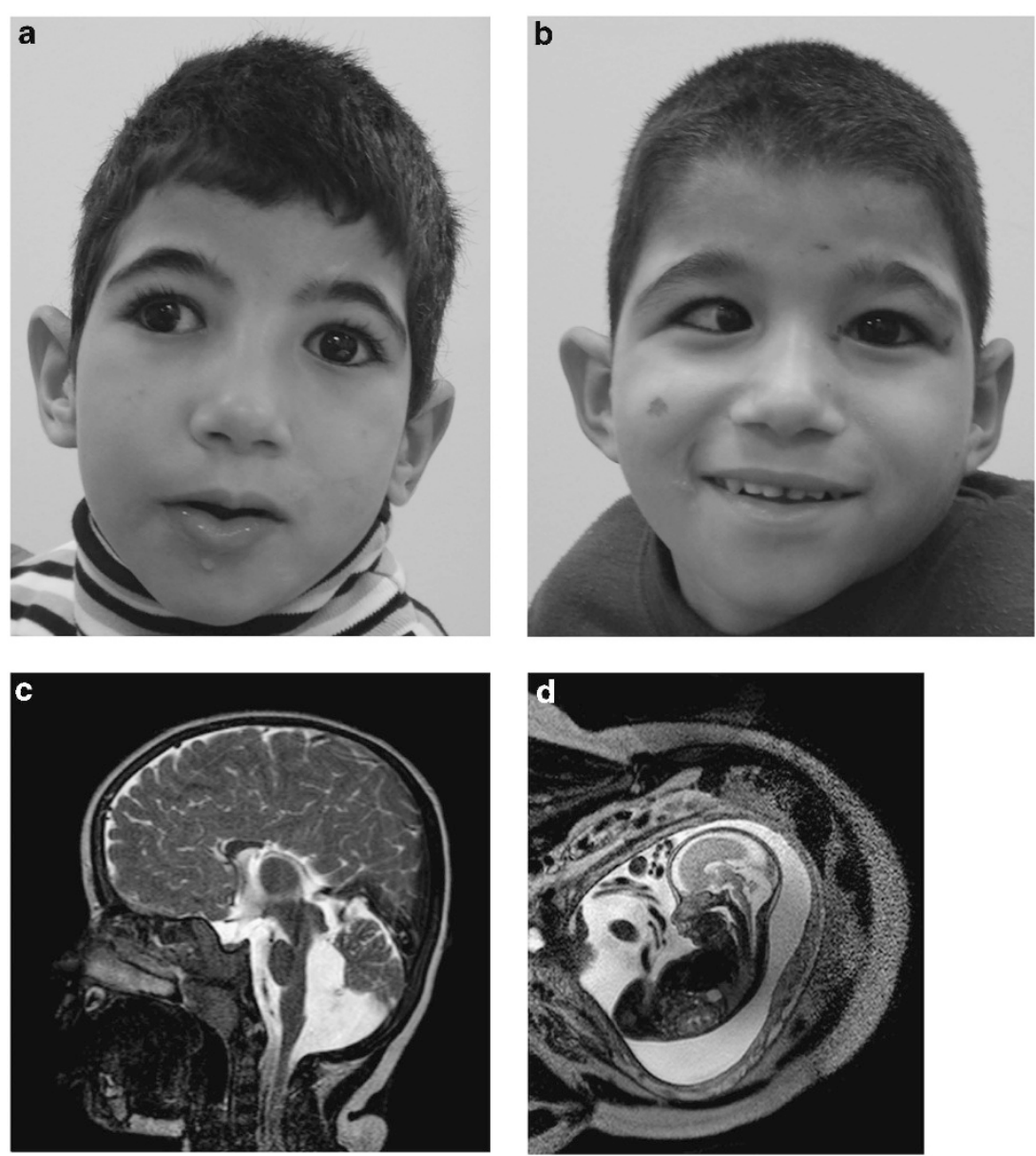

Figure 2 The disease phenotype: (a,b) Dysmorphic features seen in two of the affected individuals IV-4 at (a) and V-1 (b) at ages 5 and 9 months and 6 years, respectively. (c) Brain magnetic resonance imaging (MRI) of an affected individual V-1. Sagital T2 image demonstrates partial agenesis of corpus calosum and inferior vermis hypoplasia with moderately enlarged fourth ventricle cyst. (d) Sagital T2 fetal MRI at 25 weeks of gestation (individual IV5), showing severe hypoplasia of the vermis and cerebellum with enlarged retrocerebellar cistern.

nucleotide exchange of ARF6. ${ }^{6}$ FRMD4A binds directly to the coiledcoil domain of cytohesin1, yet has direct interaction also with the Par3 protein: amino acids 565-920 of FRMD4A directly interact with Par3 and are necessary and sufficient for its localization at primordial AJs and TJs. ${ }^{6}$ The fact that FRMD4A binds to both Par3 and the ARF6 GEF (cytohesin1) suggests that FRMD4A mediates the interaction between them and that the Par3/FRMD4A/cytohesin1 complex ensures accurate activation of ARF6 protein. ${ }^{4}$ In the predicted mutant truncated FRMD4A protein, the coiled-coil region is not affected, but serine-rich regions which are known to interact with the Par3 protein are altered: of amino acids 565-920 of FRMD4A, the predicted mutant protein ends at amino acid 740 with only 716 initial amino acids being of the original protein sequence.

The role of FRMD4A, studied in detail in epithelial cells, is of relevance also to the brain: actin cytoskeleton dynamics and membrane trafficking are tightly connected and are among the most important driving forces of neuronal development, basic synaptic transmission events and synaptic plasticity. ${ }^{8,9}$ One group of proteins involved in coordination of these two processes is the family of ADP ribosylation factors (ARFs). ${ }^{10,11}$ ARF6 is the only member of the ARF family that can simultaneously regulate actin cytoskeleton changes and membrane exchange between plasma membrane and endocytic compartments. ${ }^{8,9}$ ARF6 and its GEFs are expressed in the brain. Arf6 has been shown to modulate morphological processes crucial for proper formation of the neuronal circuits in the brain, including regulating cell polarity in neurons, dendrite and axon differentiation, development of dendritic arbor complexity and dendritic spine formation, as well as determining dendritic branching in hippocampal neurons and neurite outgrowth in PC12 cells. ${ }^{8,9}$ As seen in molecular processes determining epithelial cell polarity, ${ }^{6}$ FRMD4A has an important role in the activation of ARF6 through mediating the interaction between Par3 and the ARF6 GEF. It is thus likely that the neurological phenotype seen in the affected individuals is due to inability of the mutant FRMD4A to mediate such interaction in the absence of part of the domain that is essential for Par3 binding.

Aside from the effect on Par3 binding, it is plausible that the mutation also affects the FRMD4A-mediated interaction between GEF and ARF6: besides the FERM domain, FRMD4A contains two serinerich regions. ${ }^{6}$ Serine-rich regions can introduce a conformational change in the structure of a protein via interaction with other hydrophobic and hydrophilic residues in the protein following phosphorylation of the serine residues within these regions. ${ }^{10}$ The mutation we describe results in putative loss of one of the Ser-rich regions and the shortening of the other (Figure 3e), possibly disrupting the function of the protein through altering its conformation, likely preventing the interaction between GEF and ARF6 and thereby ARF6 activation. 
Table 1 Phenotypic details of four affected individuals (IV-3, IV-4, IV-10 and V-1 of Figure 1): HC, weight and height at different time points from birth to age 7 years

\begin{tabular}{|c|c|c|c|c|c|c|c|c|c|c|c|c|}
\hline Patient & $\begin{array}{c}\text { HC -birth } \\
(\mathrm{cm})\end{array}$ & $\begin{array}{l}\text { HC-1Year } \\
\text { (cm) }\end{array}$ & $\begin{array}{c}\text { HC-3Years } \\
(\mathrm{cm})\end{array}$ & $\begin{array}{c}\text { HC-7Years } \\
\text { (cm) }\end{array}$ & $\begin{array}{l}\text { Weight- } \\
\text { birth(kg) }\end{array}$ & $\begin{array}{l}\text { Weight- } \\
1 \text { Year (kg) }\end{array}$ & $\begin{array}{c}\text { Weight- } \\
\text { 3Years (kg) }\end{array}$ & $\begin{array}{c}\text { Weight- } \\
\text { 7Years (kg) }\end{array}$ & $\begin{array}{c}\text { Height- } \\
1 \text { Year }(\mathrm{cm})\end{array}$ & $\begin{array}{c}\text { Height- } \\
\text { 3Years }(\mathrm{cm})\end{array}$ & $\begin{array}{c}\text { Height- } \\
\text { 7Years }(\mathrm{cm})\end{array}$ & $\begin{array}{c}\text { Vineland adequacy } \\
\text { at } 3 \text { Years }\end{array}$ \\
\hline IV -3 & 30.5 & 39.5 & 44.0 & 45.5 & 2.765 & 5.1 & 10.5 & 14.5 & 65 & 87 & 105 & 5 Months \\
\hline IV -4 & 32.0 & 38.0 & 44.2 & 45.0 & 3.280 & 5.9 & ND & 19.0 & 68 & ND & 117 & 7 Months \\
\hline IV-10 & 29.0 & 39.5 & 42.5 & ND & 2.260 & 6.4 & 10.3 & ND & 68 & 87 & ND & 9 Months \\
\hline V-1 & 31.5 & 42.0 & 43.6 & ND & 2.700 & ND & 12.0 & ND & 75 & 87 & ND & 5 Months \\
\hline
\end{tabular}

All four affected individuals had undergone the Vineland adaptive behavior scale evaluation at age 3 years, demonstrating developmental capabilities appropriate for ages 5-9 months.

a

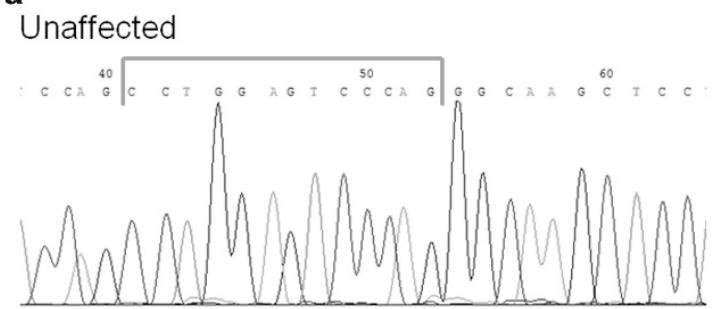

b

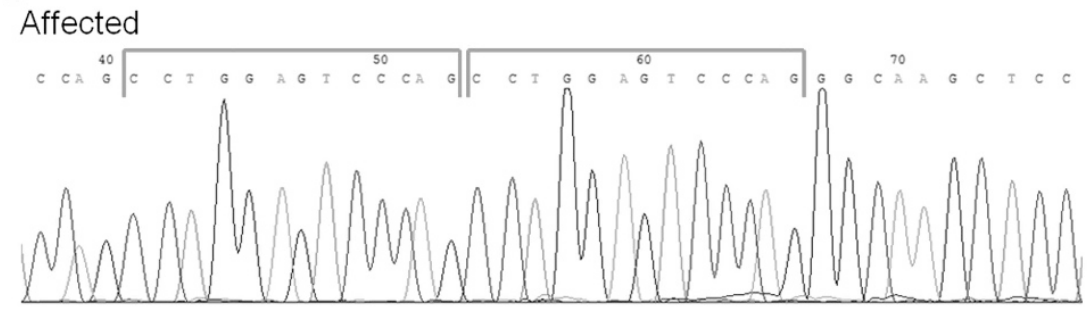

C

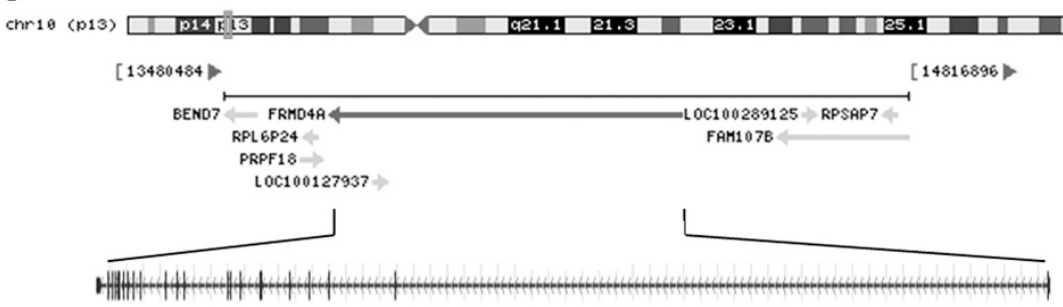

d

Normal DNA sequence

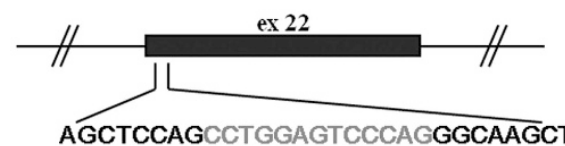

c.2133_2145dup mutation

AGCTCCAGCCTGGAGTCCCAGCCTGGAGTCCCAGGGCAAGCT

e

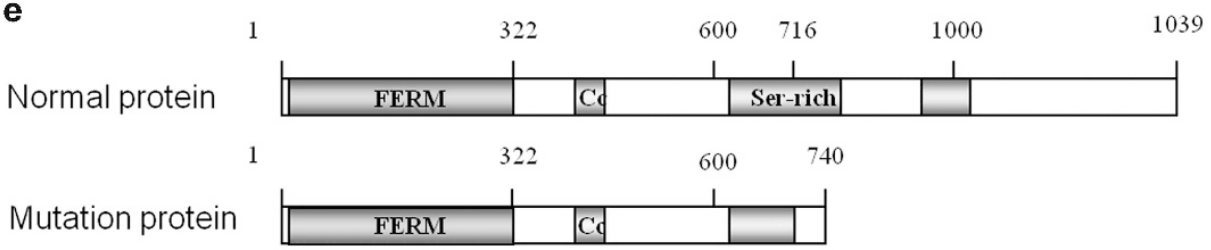

Figure 3 The 2134_2146dup13 FRMD4A mutation: Sequence analysis of an unaffected (a), and affected (b) individuals. (c) Schematic representation of the chromosomal locus and the genomic DNA of FRMD4A. (d) The homozygous c.2134_2146dup13 mutation. (e) Schematic illustration of the FRMD4A protein structure (Uniprot) and the predicted mutant truncated protein (p.Gly716Profs*25). FERM domain marked in orange, hypothetical coiled-coil region marked in yellow and Ser-rich regions marked in green. Chr, Chromosome; ex, exon; Ser, serine; Cc, coiled-coil. The full colour version of this figure is available at European Journal of Human Genetics online. 
The fact that a homozygous FRMD4A null mutation is compatible with life is likely, at least in part, due to redundancy between FRMD4A and FRMD4B: both proteins are expressed in the brain, and both are capable of direct interaction with cytohesin1 through their coiled-coil region and to the Par3 protein through their serine-rich domains. ${ }^{4}$ Moreover, FRMD4B was shown to be capable of compensating the loss of FRMD4A function in knock down cells. ${ }^{6}$

As a final note, it is of interest that a recent report suggests association of FRMD4A with Alzheimer's disease: combining both a genome-wide homozygosity association study and a conservative three-stage replication approach, FRMD4A polymorhpisms were suggested to be a risk factor for Alzheimer's disease. ${ }^{11}$ This is in line with a recent observation of an association of the FRMD4A locus with plasma $A \beta 1-42 / A \beta 1-40$, reinforcing the plausibility of the association of FRMD4A with Alzheimer's disease risk and its potential implication in a subtle control of the APP metabolism. ${ }^{112}$ Thus, FRMD4A polymorphisms are a likely risk factor for Alzheimer's disease. Our data demonstrate that a homozygous null mutation of the same gene causes a severe neurological phenotype with unique dysmorphism.

\section{CONFLICT OF INTEREST}

The authors declare no conflict of interest.

\section{ACKNOWLEDGEMENTS}

Funding for this research was provided by Teva Pharmaceutical Industries Ltd. under the Israeli National Network of Excellence in Neuroscience (NNE) established by Teva. Funding for this research was provided also by the Legacy Heritage Bio-Medical Program of the Israel Science Foundation (grant no. 1689/12) and the Kahn Family Foundation. We thank the Broad Institute for whole-exome data processing and the families for participating in the study.

\section{WEB RESOURCES}

ClustalW software: http://www.ebi.ac.uk/Tools/clustalw/DNAnexus: https://

dnanexus.com/

dbSNP database: http://www.ncbi.nlm.nih.gov/projects/SNP/

Exome Variant Server, NHLBI Exome Sequencing Project, Seattle, WA:

http://evs.gs.washington.edu/EVS/

HomoloGene database: http://www.ncbi.nlm.nih.gov/homologene

Leiden Open Variation Database: http://databases.lovd.nl/

NimbleGen SeqCap EZ Human Exome Library v2.0: http://www.nimblegen. com/products/seqcap/ez/v2/

Uniprot: http://www.uniprot.org

1 Gilmore EC, Walsh CA: Genetic causes of microcephaly and lessons from neurona development. Wiley Interdiscip Rev Dev Biol 2013; 2: 461-478.

2 Markus B, Alshafee I, Birk OS: Deciphering the fine-structure of tribal admixture in the Bedouin population using genomic data. Heredity (Edinb) 2014; 112: 182-189.

3 Zolotushko J, Flusser H, Markus B et al: The desmosterolosis phenotype: spasticity, microcephaly and micrognathia with agenesis of corpus callosum and loss of white matter. Eur J Hum Genet 2011; 19: 942-946.

4 Fishelson M, Geiger D: Exact genetic linkage computations for general pedigrees. Bioinformatics 2002; 18: S189-S198.

5 Markus B, Narkis G, Landau D et al: Autosomal recessive congenital contractural syndrome type 4 (LCCS4) caused by a mutation in MYBPC1. Hum Mutat 2012; 33 1435-1438.

6 Ikenouchi J, Umeda M: FRMD4A regulates epithelial polarity by connecting Arf6 activation with the PAR complex. Proc Nat Acad Sci 2010; 107: 748-753.

7 Tepass U: FERM proteins in animal morphogenesis. Curr Opin Genet Dev 2009; 19 357-367.

8 Hernandez-Deviez DJ, Casanova JE, Wilson JM: Regulation of dendritic development by the ARF exchange factor ARNO. Nature Neuro Sci 2002; 5: 623-624.

9 Jaworski J: ARF6 in the nervous system. Eur J Cell Biol 2007; 86: 513-524.

10 Huang Y, Steitz JA: SRprises along a messenger's journey. Mol Cell 2005; 17 613-615

11 Lambert JC, Grenier-Boley B, Harold D et al: Genome-wide haplotype association study identifies the FRMD4A gene as a risk locus for Alzheimer's disease. Mol Psych 2012 18: $461-470$

12 Sannerud R, Declerck I, Peric A, Raemaekers T et al: ADP ribosylation factor 6 (ARF6) controls amyloid precursor protein (APP) processing by mediating the endosomal sorting of BACE1. Proc Nat Acad Sci 2011; 108: E559-E568. 